\title{
Combined Intracorneal and Subconjunctival Bevacizumab Injections for Recurrent Visual Loss and Intraocular Hemorrhage from Vascularized Fibrous Downgrowth
}

\author{
Ahmad M. Mansour a, b \\ Departments of Ophthalmology, ${ }^{a}$ American University of Beirut and ${ }^{\mathrm{b}}$ Rafic Hariri \\ University Hospital, Beirut, Lebanon
}

\section{Key Words}

Epithelial downgrowth · Fibrous downgrowth $\cdot$ Intracorneal bevacizumab $\cdot$ Subconjunctival bevacizumab

\begin{abstract}
Background: Epithelial and fibrous downgrowths result from persistently leaky wounds with conjunctival or corneal epithelium and fibroblasts growing over corneal endothelium, iris and angle structures, leading to corneal edema and difficult-to-control glaucoma. Therapies have included en bloc resection, cryotherapy, endolaser or external photocoagulation and either 5-fluorouracil or mitomycin C injections. A new complication and a novel therapy are presented. Case Presentation: A 44-year-old uniocular housewife sustained a large corneoscleral laceration following a fall. She underwent primary suturing and was subsequently referred for repair of retinal detachment. She underwent resuturing of her gaping wound, pars plana vitrectomy and scleral buckle. Postoperative visual acuity was 6/120 due to a large macular hole. Visual acuity dropped to hand motion 5 times in a recurrent fashion due to diffuse intraocular hemorrhage. Vascularized fibrous downgrowth was the identifiable source of bleeding into the anterior and posterior chambers. This was controlled by 21 intracorneal and subconjunctival bevacizumab injections over 2.5 years of therapy. Conclusion: Repeated bevacizumab injections can control intraocular bleeding from new vessels accompanying fibrous downgrowth with preservation of vision.
\end{abstract}


Mansour: Intracorneal and Subconjunctival Bevacizumab Injections for Recurrent

\section{Background}

Epithelial and fibrous downgrowths are rare, yet potentially devastating, complications of penetrating ocular trauma and ophthalmic surgery. The incidence appears to be declining with modern surgical techniques [1]. Various clinical and laboratory investigations have emphasized the complexity of managing these cases and the poor visual prognosis [1-11]. Current therapies have included en bloc dissection [3] of the membrane, cryotherapy or endophotocoagulation [4] of the sheet. We present a novel therapy in a uniocular subject with recurrent visual loss due to repeated intraocular bleeding.

\section{Case Presentation}

This 44-year-old housewife sustained ocular injuries 23 years ago after a motor vehicle accident and was operated bilaterally. Visual acuity was since lost in the right eye and the left eye was maintained on ocular hypotensive drops. Two weeks before presentation, she had fallen from a ladder and had sustained extensive corneoscleral laceration of the left eye with uveal prolapse and total hyphema. The laceration was sutured in an outside hospital.

Visual acuity on presentation was light perception with through-and-through vertical corneal wound, dense hyphema and large inferior conjunctival bleb. Intraocular pressure was $5 \mathrm{~mm} \mathrm{Hg}$. B-scan ultrasonography revealed total retinal detachment. The corneoscleral wound was resutured because it was gaping. A scleral buckle was placed at $360^{\circ}$. There was profuse continuous bleeding from the iris and ciliary body area throughout surgery despite pars plana cleanout of the anterior segment (lens remnant, vitreous and blood clot). During posterior vitrectomy, a one-disc diameter macular hole was suspected. Silicone oil fill of the vitreous cavity was followed by endolaser of an extensive inferior giant retinal tear. Postoperatively, the patient had multiple sessions of laser retinopexy to seal a localized inferior retinal detachment. A large retrocorneal membrane was evident 6 weeks postoperatively over the temporal cornea (fig. 1). Postoperative best spectacle-corrected visual acuity was 6/60. A rise in intraocular pressure was again controlled by topical $\beta$-blocker. She had 5 recurrent episodes of visual loss to the level of hand motion from intraocular bleeding (interface hemorrhage, hyphema and anterior vitreous hemorrhage) (fig. 2). Visual loss used to resolve 1 week after subconjunctival $(0.1 \mathrm{ml} ; 2.5 \mathrm{mg}$ ) and intracorneal $(0.05 \mathrm{ml} ; 1.25 \mathrm{mg})$ bevacizumab (after formal consent). The rationale was the vascular nature of the fibrovascular membrane with feeder vessels consisting of several branches of limbal and corneal stromal neovascularization (fig. 3). The injections were done in a sterile fashion using a speculum and a 30-gauge needle. The intrastromal (corneal) and subconjunctival injections were aimed at the site of the feeder vessels (fig. 3). We avoided intracameral injections because of the concomitant presence of glaucoma and intravitreal silicone oil. The patient was instructed to have monthly injections. When she was late for follow-up, intraocular bleeding would occur. The patient was followed for 40 months after presentation with a final vision of $6 / 120$. She received a total of 21 injections over a period of 2.5 years with a total of 5 episodes of vitreous hemorrhage.

Written informed consent was obtained from the patient for publication of this case report and any accompanying images. A copy of the written consent is available for review by the Editor-in-Chief of this journal. 
Mansour: Intracorneal and Subconjunctival Bevacizumab Injections for Recurrent

\section{Discussion}

Epithelial and fibrous downgrowths represent migration of corneal or conjunctival epithelial or fibroblast tissue inside the eye. The most common presenting sign is a retrocorneal membrane that appears as a grayish sheet attached to the endothelium growing circumferentially, then centrally and occasionally growing over the iris with distortion of the stromal surface [1]. While some cases may exhibit subtle clinical changes mimicking iritis, other cases show extensive fibrous downgrowth like in the present case. Retrocorneal membranes arise from 1 of 3 mechanisms: epithelial downgrowth (ingrowth), fibroblastic or stromal (keratocytic) downgrowth, fibrous metaplasia of the corneal endothelium or a combination thereof [7]. Experimental studies [6] identified 3 factors that promote fibrous downgrowth: healthy keratocytes, large Descemet's break and damaged endothelium surrounding a wound. Theories to explain epithelial or fibrous downgrowth included: (1) implantation of epithelial cells; (2) incorporation of a conjunctival flap through a wound and (3) delayed closure of a corneoscleral wound [1, 2, 8]. This third mechanism is the most likely explanation with migrating epithelial and stromal keratocytes gaining entry through a persistently open wound. In the present case, retrocorneal fibrous membrane developed in a patient with a severely traumatized eye owing to a combination of preexisting pathologic conditions (gaping extensive wound involving the limbus), prolonged inflammation (vitreous, lens and hemorrhage admixture) and dense intraocular hemorrhage. Küchle and Green [2] found a multilayer of surface epithelium growing on intraocular surfaces such as the cornea, iris, chamber angle and ciliary body. Bloomfield et al. [6] detected a double-layered retrocorneal fibrous membrane, produced in part by a vascularized fibrous ingrowth. Immunohistochemistry can identify different provenances reliably in retrocorneal membranes [7].

Complications associated with epithelial and fibrous downgrowth include pupillary block, secondary glaucoma, iridocyclitis, corneal edema, corneal decompensation, loss of vision and intractable pain. Glaucoma is the most common complication resulting from epithelial downgrowth over the angle [1]. Recurrent intraocular hemorrhage is added as a newly reported complication of fibrous downgrowth. Interface hemorrhage was also reported between the cornea and the retrocorneal membranes by Chandler et al. [9]. In the present case, uncontrollable intraocular bleeding was noted during vitrectomy, which in retrospect stemmed from the retrocorneal membrane. A similar case of retrocorneal membrane causing postsurgical hyphema after cataract surgery was described by Bloomfield and Jakobiec [6]. The feeder vessels of the retrocorneal membrane in the current case stemmed from the limbal corneal neovascularization. However, we cannot rule out the possibility of concomitant feeder vessels from the ciliary body or iris. Chandler et al. [9] previously noted interface bleeding between the cornea and the retrocorneal membrane.

Fibrous ingrowth (also termed stromal downgrowth or retrocorneal membrane) shares the same risk factors as epithelial ingrowth; however, it is different in several respects: (1) vascularization occurs exclusively in fibrous membranes with an important role for growth factors like connective tissue growth factor [10] and vascular endothelial growth factor; (2) the fibrous component is predominant in fibrous downgrowth, while the cellular component is predominant in epithelial ingrowth; (3) unlike in epithelial downgrowth, there are no adjunctive tests to confirm the diagnosis of stromal downgrowth [1] and (4) it has a relatively more benign clinical course, and if surgical intervention is required, removal of all the fibrous proliferations is not required as it is the case in epithelial downgrowth [1].

Irradiation was initially tried in the last century to treat epithelial or fibrous downgrowth with poor success. Other treatment modalities involved surgical scraping [1], 
Mansour: Intracorneal and Subconjunctival Bevacizumab Injections for Recurrent

peeling, alcohol treatment, diathermy, cryotherapy [1], photocoagulation [4] and wide excision of the epithelial proliferation with ablative therapy to adjacent structures in order to eliminate residual cells. Unfortunately, these modalities were associated with a high failure rate related to difficulty in identifying the borders of the lesion and the destructive nature of the surgical procedures ultimately leading to phthisis and enucleation. In a few centers with vast experience in dealing with epithelial ingrowth, en bloc excision [3] consisted of simultaneous removal of epithelial ingrowth together with the adjacent iris, pars plicata, cornea and sclera. The resulting defect was covered with a tectonic corneoscleral graft with no clinical evidence of recurrence of epithelial or fibrous ingrowth. The main postoperative complications with this technique were vitreous hemorrhage and corneal endothelial decompensation [3]. More recently, intracameral antimetabolites like 5fluorouracil and mitomycin C have been used with some success [11].

The use of bevacizumab to cause regression of the new vessels inside the fibrovascular sheet has not been described before in fibrous downgrowth. Bevacizumab, a full-length immunoglobulin, has a $12 \mathrm{~nm}$ long, Y-shaped configuration with a molecular weight of 149 $\mathrm{kDa}$. Its 3 arms are rods approximately $3.5 \mathrm{~nm}$ in diameter. Intrastromal corneal injection of bevacizumab in rabbits could achieve an elevated level of the immunoglobulin in the ciliary body [12]. Also subconjunctival injection was found to be a reliable administration route for bevacizumab with the immunoglobulin still present in the corneal stroma 21 days after a single subconjunctival injection in mice [13]. The need for a continuous control of these new vessels by monthly injections appears similar to the requirement in diabetic fibrovascular membranes and choroidal new vessels in age-related macular degeneration. Corneal injections of bevacizumab have been administered in corneal new vessel formation [14]. Fibrovascular ingrowth appears similar in response to bevacizumab to diabetic fibrovascular membranes. The end point in the current treatment is to stop further growth of the vascularized fibrous sheet inducing regression of the ingrowth vessels and hence reduction of intraocular bleeding.

In conclusion, vascular fibrous ingrowth is thought to have the vascular leading edge as the spearhead driving the sheet inside the eye. Therapies aiming at regression of these new vessels can halt progression of the ingrowth by decreasing supply of nutrients to that tissue. Current therapies aim at surgical resection of the ingrowth or shrinkage of the fibrous element by laser therapy or chemotherapy. The current case emphasizes the role of bevacizumab in controlling vascularized fibrous ingrowth and in the resolution of intraocular hemorrhage.

\section{Disclosure Statement}

The author declares that he has no competing interests.

\section{References}

1 Chen SH, Pineda R 2nd: Epithelial and fibrous downgrowth: mechanisms of disease. Ophthalmol Clin North Am 2002;15:41-48.

2 Küchle M, Green WR: Epithelial ingrowth: a study of 207 histopathologically proven cases. Ger J Ophthalmol 1996;5:211-223.

-3 Naumann GO, Rummelt V: Block excision of cystic and diffuse epithelial ingrowth of the anterior chamber. Report on 32 consecutive patients. Arch Ophthalmol 1992;110:223-227.

4 Jadav DS, Rylander NR, Vold SD, Fulcher SF, Rosa RH Jr: Endoscopic photocoagulation in the management of epithelial downgrowth. Cornea 2008;27:601-604. 


\begin{tabular}{l|l}
\hline Case Rep Ophthalmol 2014;5:28-33 & \\
\hline DOI: 10.1159/000358167 & $\begin{array}{l}\text { ○ 2014 S. Karger AG, Basel } \\
\text { www.karger.com/cop }\end{array}$ \\
\hline
\end{tabular}

Mansour: Intracorneal and Subconjunctival Bevacizumab Injections for Recurrent Visual Loss and Intraocular Hemorrhage from Vascularized Fibrous Downgrowth

5 Shaikh AA, Damji KF, Mintsioulis G, Gupta SK, Kertes PJ: Bilateral epithelial downgrowth managed in one eye with intraocular 5-fluorouracil. Arch Ophthalmol 2002;120:1396-1398.

-6 Bloomfield SE, Jakobiec FA, Iwamoto T: Fibrous ingrowth with retrocorneal membrane. Ophthalmology 1981;88:459-465.

7 Jakobiec FA, Bhat P: Retrocorneal membranes: a comparative immunohistochemical analysis of keratocytic, endothelial, and epithelial origins. Am J Ophthalmol 2010;150:230-242.

8 Sherrard ES, Rycroft PV: Retrocorneal membranes. II. Factors influencing their growth. Br J Ophthalmol 1967;51:387-393.

-9 Chandler JW, Sugar J, Kaufman HE: Hemorrhages in the interface between corneal grafts and retrocorneal membranes. Arch Ophthalmol 1974;92:299-300.

-10 Wunderlich K, Senn BC, Reiser P, Pech M, Flammer J, Meyer P: Connective tissue growth factor in retrocorneal membranes and corneal scars. Ophthalmologica 2000;214:341-346.

-11 Yu CS, Chiu SI, Tse RKK: Treatment of cystic epithelial downgrowth with intralesional administration of mitomycin C. Cornea 2005;24:884-886.

12 Nomoto H, Shiraga F, Kuno N, Kimura E, Fujii S, Shinomiya K, Nugent AK, Hirooka K, Baba T: Pharmacokinetics of bevacizumab after topical, subconjunctival, and intravitreal administration in rabbits. Invest Ophthalmol Vis Sci 2009;50:4807-4813.

13 Dastjerdi MH, Sadrai Z, Saban DR, Zhang Q, Dana R: Corneal penetration of topical and subconjunctival bevacizumab. Invest Ophthalmol Vis Sci 2011;52:8718-8723.

14 Mohammadpour M: Deep intrastromal injection of bevacizumab for the management of corneal neovascularization. Cornea 2013;32:109-110.

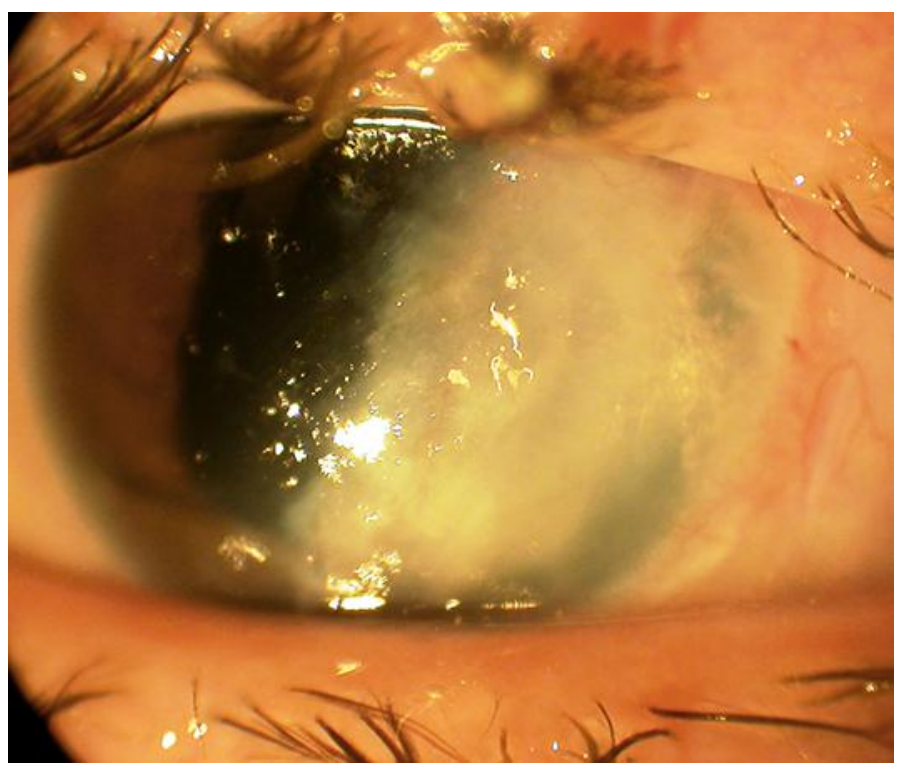

Fig. 1. Slit-lamp photography shows the central vertical corneal wound with retrocorneal fibrous downgrowth covering the temporal half of the cornea, as evident 6 weeks after wound revision and vitreoretinal surgery. 
Case Reports in

Ophthalmology
Case Rep Ophthalmol 2014;5:28-33

DOI: $10.1159 / 000358167$

Mansour: Intracorneal and Subconjunctival Bevacizumab Injections for Recurrent

Visual Loss and Intraocular Hemorrhage from Vascularized Fibrous Downgrowth

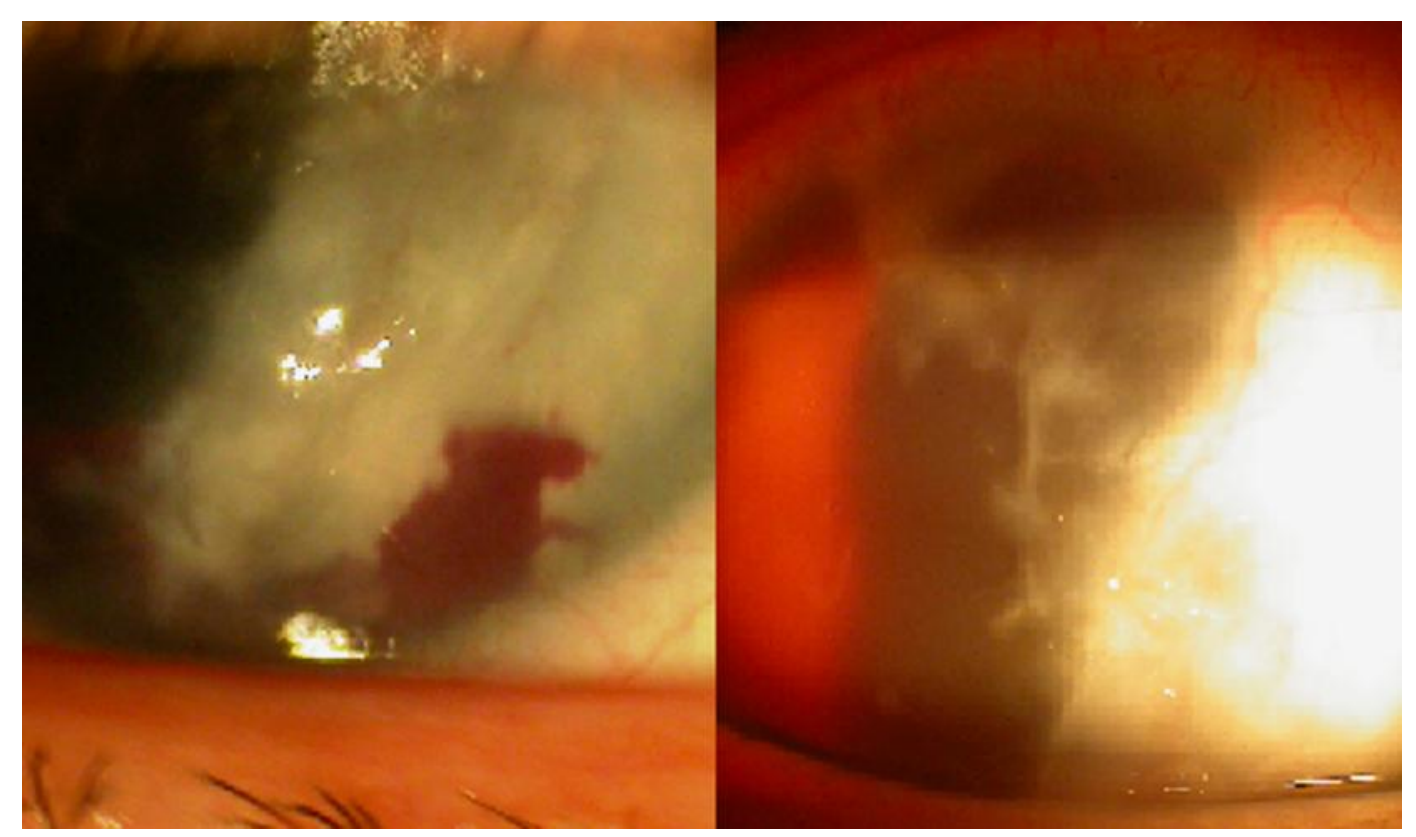

Fig. 2. Left: streak of retrocorneal interface hemorrhage. Right: hyphema with anterior vitreous hemorrhage in front of the silicone oil bubble resulting in hand motion vision.
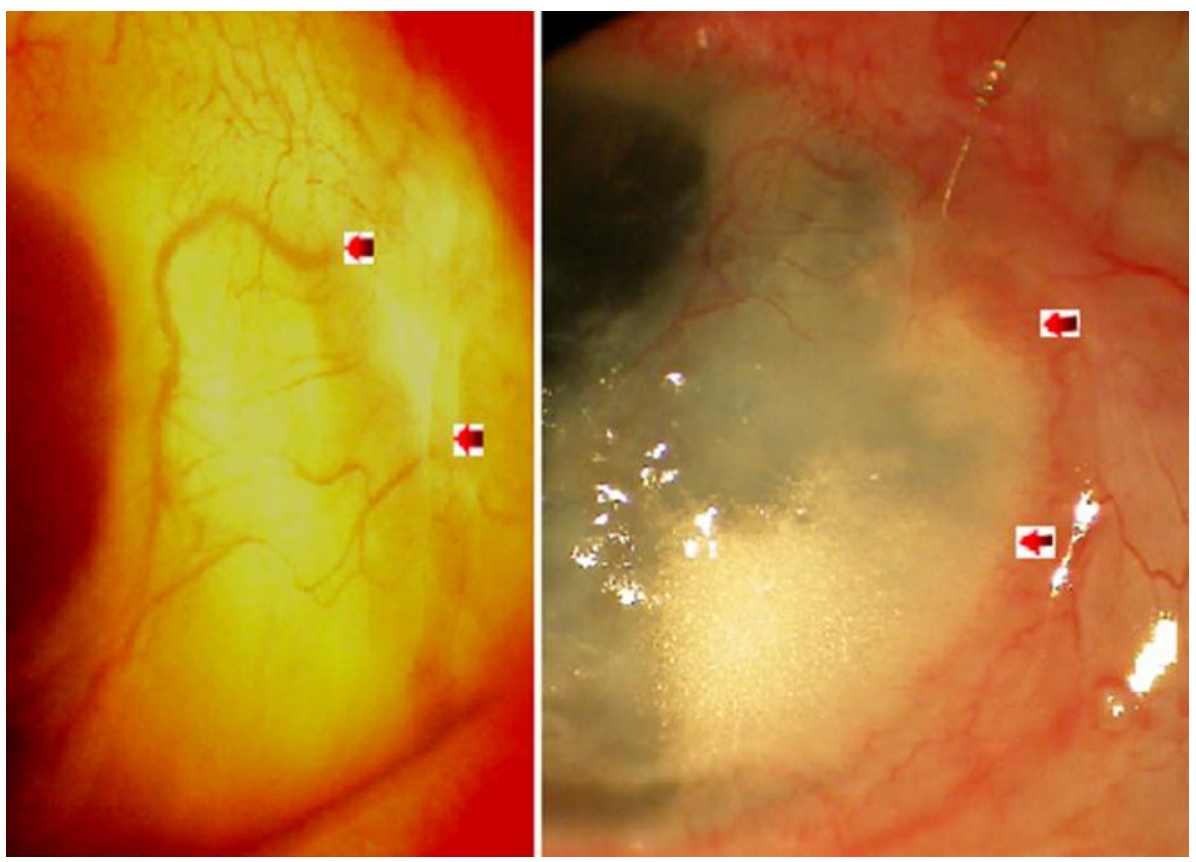

Fig. 3. Left: two feeder vessels (arrows) are noted at the temporal limbus. Subconjunctival injections are made around these two feeder vessels. Right: slit-lamp appearance after intrastromal (two arrows) injections of bevacizumab. 